\title{
Relation between branching patterns and perfusion in stochastic generated coronary arterial trees
}

\author{
J. Dankelman · A. J. M. Cornelissen • \\ J. Lagro · E. VanBavel · J. A. E. Spaan
}

Received: 6 February 2006/ Accepted: 29 November 2006/ Published online: 3 January 2007

(C) International Federation for Medical and Biological Engineering 2007

\begin{abstract}
Biological variation in branching patterns is likely to affect perfusion of tissue. To assess the fundamental consequences of branching characteristics, 50 stochastic asymmetrical coronary trees and one nonstochastic symmetrical branching tree were generated. In the stochastic trees, area growth, $A$, at branching points was varied: $A=$ random; $1.00 ; 1.10 ; 1.13$ and 1.15 and symmetry, $S$, was varied: $S=$ random; 1.00 ; $0.70 ; 0.58 ; 0.50$ and 0.48 . With random $S$ and $A$ values, a large variation in flow and volume was found, linearly related to the number of vessels in the trees. Large $A$ values resulted in high number of vessels and high flow and volume values, indicating vessels connected in parallel. Lowering symmetry values increased the number of vessels, however, without changing flow, indicating a dominant connection of vessels in series. Both large $A$ and small $S$ values gave more realistic gradual pressure drops compared to the symmetrical non-stochastic branching tree. This study showed large variations in tree realizations, which may reflect real
\end{abstract}

This work is supported by The Netherlands Heart Foundation Grant 95020 to Dr. J. Dankelman.

J. Dankelman $(\bowtie)$. A. J. M. Cornelissen .

J. Lagro

Department of BioMechanical Engineering,

Faculty of Mechanical, Maritime and Material Sciences

(3mE), Delft University of Technology, Mekelweg 2,

2628 CD Delft, The Netherlands

e-mail: j.dankelman@tudelft.nl

E. VanBavel · J. A. E. Spaan

Department of Medical Physics, Cardiovascular Research

Institute Amsterdam, Academic Medical Center,

University of Amsterdam, PO Box 22700,

1100 DE Amsterdam, The Netherlands biological variations in tree anatomies. Furthermore, perfusion of tissue clearly depends on the branching rules applied.

Keywords Coronary circulation - Arterial tree reconstruction - Branching characteristics .

Area growth · Coronary perfusion

\section{Introduction}

The architecture of the arterial tree forms a dominant factor in the distribution of blood flow. During vascular development, vascular networks remodel into complex networks through dilatation, sprouting, and bridging $[5,6,13,15,17]$. The large variability in myocardial flow per $100 \mathrm{~g}$ tissue that is found indicates variation in anatomical characteristics of the coronary arterial tree [7, 16]. Recent studies visualized the anatomical structure of the coronary arterial tree in the intact heart [10,14]. Rodriguez-Porcel et al. [10] found that myocardial architecture was altered in experimental hypercholesterolemia; hypercholesterolemia increased sub-endocardial microvascular density and sprouting. Experiments relating anatomical characteristics to perfusion are, however, still difficult to perform. Therefore, in this study a model approach is used to relate anatomical characteristics to flow, vascular volume and pressure distribution in the tree.

An exact reconstruction of the coronary arterial vascular tree over many orders of branches is not yet available. Kassab et al. [6] and VanBavel and Spaan [15] quantified coronary arterial branching patterns. Kassab et al. measured more segments than VanBavel and Spaan, however, Kassab's classification method, 
based on a modification of Strahler ordering based on diameter (diameter defined Strahler ordering) and a connectivity matrix, was difficult to use for generating trees in our simulation structure. Therefore, in this study vascular trees were generated applying VanBavel's model [15] based on measured heterogeneously branching patterns of the coronary vascular bed. Stochastic relations for length of segments, area ratio and symmetry of nodes along the bed were used to reconstruct stochastic asymmetrical branching arterial trees. The symmetry of branching patterns and area growth at branching points were varied and their influence on flow, volume and pressure distribution, and tree characteristics (maximum number of generations and Strahler order) were determined. Model studies often simplify trees by assuming symmetrical branching. Therefore, the implications of such a simplification were evaluated by comparing the results of the stochastic asymmetrical trees to the results of a non-stochastic symmetrical branching arterial tree.

\section{Methods}

2.1 Data of vascular casts used to define the coronary arterial tree

To quantify and to reconstruct the arterial tree, the morphometric data of VanBavel and Spaan [15] were used. In summary, in that study two male pig hearts have been dilated by $10 \mu \mathrm{M}$ adenosine and before supplying the casting material, the vascular bed was perfused with fixation solution at $90 \mathrm{mmHg}$ [15]. After removal of the surrounding tissue, diameter and length of 2,366 smaller vessels were measured. Figure 1 summarizes these data. Figure 1a shows the length $(L)$ versus diameter $(D)$ of these segments. A segment is defined as a part of a vessel between two nodes. From a total of 1,663 nodes, the diameters of the mother segment $\left(D_{\mathrm{M}}\right)$ and both the larger $\left(D_{\mathrm{L}}\right)$ and smaller $\left(D_{\mathrm{S}}\right)$ daughter segments were measured. The relation between mother and daughter diameters at a node was quantified by the area ratio: $A=\left(D_{\mathrm{L}}^{2}+D_{\mathrm{S}}^{2}\right) / D_{\mathrm{M}}^{2}$ and symmetry: $S=D_{\mathrm{S}} / D_{\mathrm{L}}$. The area relation $A$ and symmetry $S$ as functions of $D_{\mathrm{M}}$ are depicted in Fig. 1c and e.

\subsection{Fitting and quantification of the data}

A linear regression line, fitting the diameter data of Fig. 1a is described by $\bar{L}=10.2 \cdot D^{0.72}$, where $\bar{L}$ is the least squared estimated length in $\mu \mathrm{m}$ and $D$ the diameter in $\mu \mathrm{m}$. The distribution of the ratio between observed lengths $(L)$ and their least-squares estimates $(\bar{L})$ is given in Fig. 1b. The length distribution is quantified by: $L=\bar{L} \cdot 10^{x}$ where $x$ is gamma distributed according to:

$f_{x}(x)=\frac{(-x-\gamma)^{\alpha-1} \mathrm{e}^{\frac{x+\gamma}{\beta}}}{\beta^{\alpha} \Gamma(\alpha)}$

with $\alpha=6, \beta=0.15$ and $\gamma=-0.9$.

The linear regression line fitting the area ratio $A$ given in Fig. 1c is given by $\bar{A}=1.279-$ $0.086 .{ }^{10} \log \left(D_{\mathrm{M}}\right)$, where $\bar{A}$ is the least square estimated area ratio. The histogram of the deviations of $A$ from the regression line, depicted in Fig. 1d, is described by $A=\bar{A}+y$, with $y$ normally distributed according to:

$f_{y}(y)=\frac{1}{\sigma \sqrt{2 \pi}} \mathrm{e}^{\frac{1}{2}\left(\frac{y-\mu}{\sigma}\right)^{2}} \quad-0.8<y<0.8$

$f_{y}(y)=0 \quad y \leq-0.8 \cup y \geq 0.8$,

with $\mu=0$ and $\sigma=0.2$.

The symmetry $S$ of arterial nodes depicted in Fig. 1e is very variable. The line in the figure depicts the lower boundary of the symmetry $\left(S_{\mathrm{LB}}\right)$ as function of $D_{\mathrm{M}}$ and is described by:

$$
\begin{aligned}
S_{\mathrm{LB}} & =\frac{5}{D_{\mathrm{M}}} & & D_{\mathrm{M}}<50 \mu \mathrm{m} \\
S_{\mathrm{LB}} & =0.1 & & D_{\mathrm{M}} \geq 50 \mu \mathrm{m}
\end{aligned}
$$

The upper boundary of the symmetry is by definition unity. For symmetry a clear lower band exists because vessels smaller than $5 \mu \mathrm{m}$ do not exist. The distribution for symmetry between the lower and upper boundary, depicted in Fig. 1f, is described by $S=z$, with $z$ uniform distributed between the boundaries according to:

$$
\begin{array}{ll}
f_{z}(z)=\frac{1}{1-S_{\mathrm{LB}}} \quad & S_{\mathrm{LB}}<z<1 \\
f_{z}(z)=0 & z \leq S_{\mathrm{LB}} \cup z \geq 1
\end{array}
$$

\subsection{Mathematical reconstruction of the coronary arterial tree}

The mathematical reconstruction of the coronary arterial tree is based on the distributions of segmental length $(L)$, nodal area expansion $(A)$, and symmetry $(S)$ as function of mother diameter $\left(D_{\mathrm{M}}\right)$ (Fig. 1). Each reconstruction started with one segment with $D_{\mathrm{M}}=500 \mu \mathrm{m}$. Based on this diameter, a length $(L)$ was randomly chosen using the fitted curves in Fig. 1a 
Fig. 1 Data of vascular casts used in the model. Left panels: a length distribution of 2,366 vascular segments; c area expansion ratio and $\mathbf{e}$ symmetry of 1,663 nodes, plotted against the diameter of their mother segment on a semi-logarithmic scale. The lines in panels $\mathbf{a}$ and $\mathbf{c}$ represent the least-squares fits. The line in e represents the lower bound of symmetry as function of the diameter. Right panels: b histogram of the ratios of the observed length to their least-squared estimates on a semilogarithmic scale. The line is describing this histogram with a gamma distribution. d

Histogram of the deviations of the observed area ratio to their least squared estimates. The line is describing this histogram between -0.8 and 0.8 with a normal distribution. f Histogram of the observed symmetry between the lower bound and unity. The line is describing this histogram with a uniform distribution
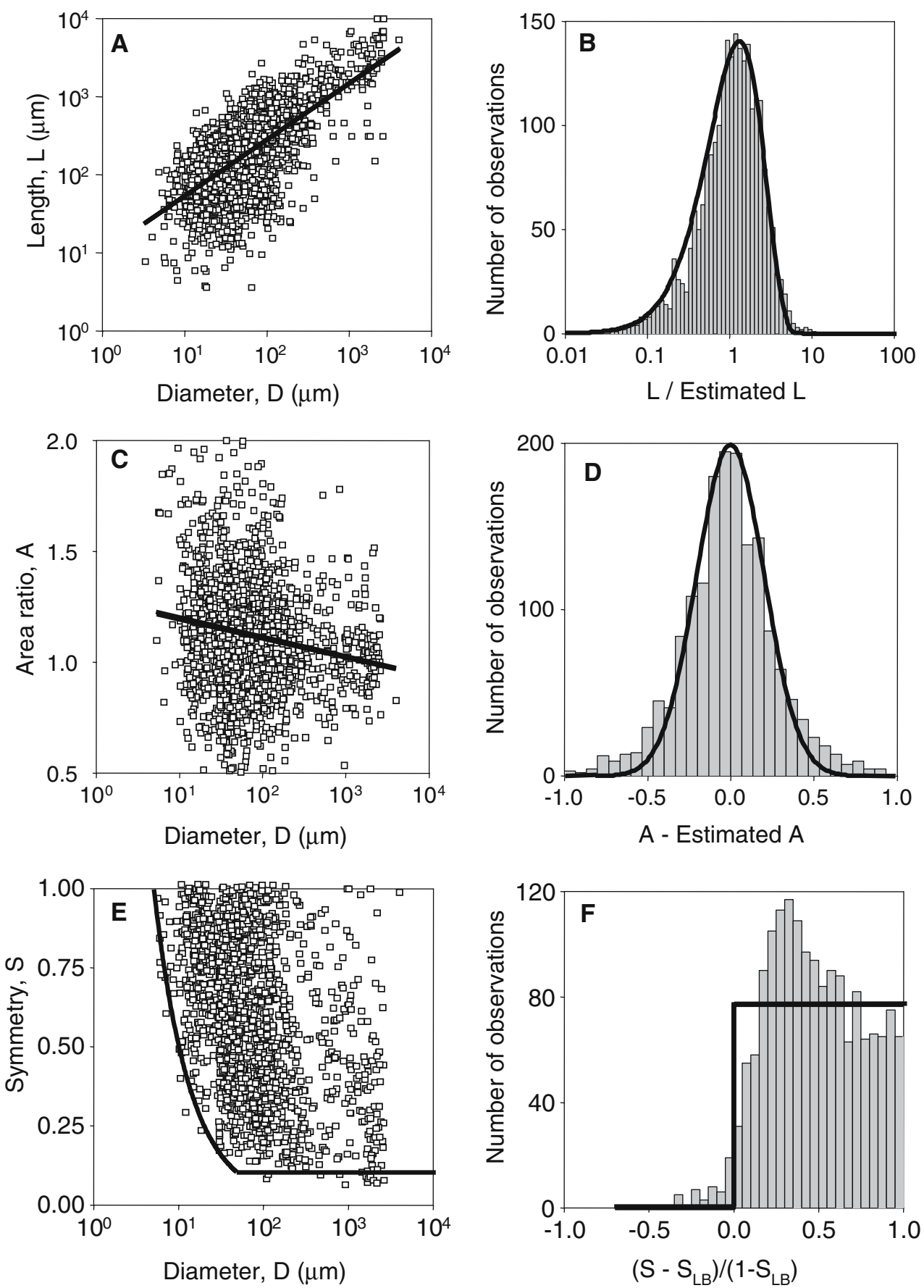

and b. Subsequently, two daughter segments were generated. Based on the mother diameter, a value for the area ratio $(A)$ was randomly chosen according to fits in Fig. 1c and d. Finally, the value for symmetry $(S)$ was randomly chosen according to the fits in Fig. 1e and $\mathrm{f}$. From the values of both $A$ and $S$, the two daughter diameters $D_{\mathrm{L}}$ and $D_{\mathrm{S}}$ were calculated. Lengths of these segments were again generated using the fitted data of Fig. 1a and b. Distal to each of these segments, two new segments were generated. This process was continued until segments with diameters $<15 \mu \mathrm{m}$ were generated.

Apart from the asymmetrical stochastic trees also a symmetrical non-stochastic branching tree was generated. The reconstruction started again with a segment with $D_{\mathrm{M}}=500 \mu \mathrm{m}$. The length and area ratio were chosen based on the least squares estimates (Fig. 1a and c). Symmetry was taken as unity. This process was continued until segments with diameters $<15 \mu \mathrm{m}$ were generated. 


\subsection{Model simulations}

The Reynolds numbers in the vessels segments in low, therefore the resistance of a vessel segment was calculated assuming flow according to Poiseuille:

$R_{i}=\frac{128 \cdot \eta_{i} \cdot L_{i}}{\pi \cdot d_{i}^{4}}$

with $L_{i}$ the length of the vessel segment $i, d_{i}$ the diameter of the vessel segment and $\eta_{i}$ the viscosity of the perfusate. The viscosity is dependent on the diameter of the vessel as determined in the rat mesentery vasculature by Pries et al. [9], assuming constant systemic hematocrit of $45 \%$ :

$$
\begin{aligned}
\eta_{i}= & {\left[1+\left(1.6 \cdot 10^{-3}\left(6 \mathrm{e}^{-0.085 d_{i}}+3.2-2.44 \mathrm{e}^{-0.06 d_{i}^{0.645}}\right)-1\right)\right.} \\
& \left.\times\left(\frac{d_{i}}{d_{i}-1.1}\right)^{2}\right]\left(\frac{d_{i}}{d_{i}-1.1}\right)^{2}
\end{aligned}
$$

Simulations were performed with an inlet pressure of $90 \mathrm{mmHg}$ and an outlet (pre-capillary) pressure of $30 \mathrm{mmHg}$ representing capillary inlet pressure. To calculate the pressure drop over each segment and the blood flow through each segment, the replacement resistance of each segment was determined. The replacement resistance of a segment $i$ is defined as the total resistance of the subtree originating in segment $i$. The smallest precapillary segments with diameters $<15 \mu \mathrm{m}$ do not have subtrees and their replacement resistance equals their segmental resistance. Starting with these segments, the replacement resistance of the whole tree was calculated according to:

$R_{\mathrm{sub}, i}=R_{i}+\left(\frac{1}{R_{\mathrm{sub}, i, \mathrm{~S}}}+\frac{1}{R_{\mathrm{sub}, i, \mathrm{~L}}}\right)^{-1}$

with $R_{\mathrm{sub}, i}$ the replacement resistance of segment $i ; R_{i}$ the resistance of the segment; $R_{\mathrm{sub}, i, \mathrm{~S}}$ and $R_{\mathrm{sub}, i, \mathrm{~L}}$ the replacement resistances of the small and large daughters of segment $i$, respectively.

All tree generations and calculations were performed in $\mathrm{C}++$. In the first simulations, a set of 50 stochastic asymmetrical branching trees and one nonstochastic symmetrical branching tree were generated. Additional sets of trees were generated to investigate the impact of choices for $A$ and $S$ at branching points. We assumed various constant values for either $A$ or $S$. For each constant $S$ value, a set of 50 trees was generated with randomly chosen $A$ values and for each constant $A$ value, a set of 50 trees with randomly chosen $S$ values. Due to simulation limitations $S$ and $A$ values were varied such that $N_{\text {segments }}$ of the trees were $<200,000$. Five different $S$ values were considered: $S=1,0.70,0.58,0.5$ and 0.48 , with $S=0.58$ being the average $S$ value of all vessels presented in Fig. 1e. Four different values for $A$ were considered: $A=1,1.1,1.13$ and 1.15 for all diameters, with $A=1.13$ being the average $A$ value of the vessels presented in Fig. 1c.

\subsection{Data analysis and presentation}

Generated trees were characterized by their total number of segments $\left(N_{\text {segments }}\right)$, inflow, vascular volume, maximum number of generations $\left(N_{\text {gen }}\right.$, i.e. the maximum number of series-arranged segments), and maximum number of Strahler orders $\left(N_{\text {strahler }}\right)$. For each set of trees (i.e. fully random, random $A$ or random $S$ ), these parameters were averaged over the 50 trees. The averages of the sets were compared by ANOVA. Due to the stochastic nature of the branching rules, the 50 generated trees within a set will all be different. In particular, they will have different sizes, as quantified by $N_{\text {segments }}$. To test whether for a given set of trees inflow, volume, $N_{\text {gen }}$ and $N_{\text {strahler }}$ depend on $N_{\text {segments, }}$, linear regression analysis was performed for the 50 realizations within each tree set. These regressions were then compared for the various sets using ANOVA.

To analyze the pressure profile along the networks, the segments in the stochastic trees were classified in 15 diameter classes. For each individual tree, pressures of all segments in the 15 classes were averaged. These values were then used to calculate mean and standard deviation of the pressure profile over the 50 realizations within a tree set. The impact of $S$ and $A$ on these pressure profiles was only graphically analyzed.

\section{Results}

\subsection{Flow and vascular volume in reconstructed} trees

Total inflow and total vascular volume values of 50 stochastic asymmetrical trees with $A$ and $S$ random (circle) and the non-stochastic symmetrical branching tree (asterisks) are provided as function of $N_{\text {segments }}$ in Fig. $2 \mathrm{a}$ and b. Inflow and vascular volume are both strongly correlated to $N_{\text {segments }}\left(r^{2}>0.95\right.$, first row Table 1). 
Fig. 2 Simulation results of 50 stochastic asymetrical trees and 1 symmetrical branching tree. a Total inflow and $\mathbf{b}$ total arterial volume of the reconstructed trees, given as a function of $N_{\text {segments }}$ in the trees. c Maximum number of generations and d Strahler order. Circle: 50 asymmetrical stochastic branching trees; asterisks: symmetrical branching tree
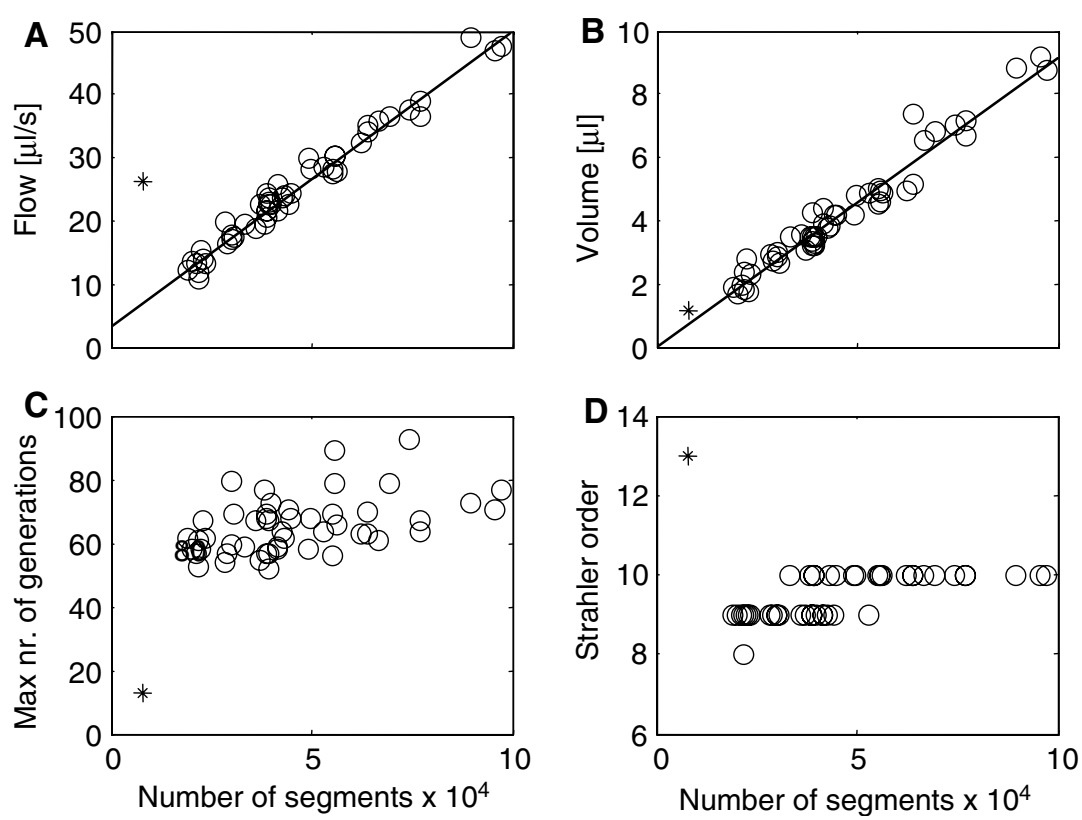

Table 1 Number of segments, flow and volume values (mean \pm SD) and slopes and $r^{2}$ of regression lines

\begin{tabular}{|c|c|c|c|c|c|c|c|c|}
\hline$S$ & $A$ & $N_{\text {segments }}$ & Flow $(\mu \mathrm{l} / \mathrm{s})$ & Volume $(\mu \mathrm{l})$ & $\begin{array}{l}\text { Slope } \\
\left(N_{\text {seg }}-\right. \\
\text { flow } \times 10^{-4} \\
(\mu 1 / s)\end{array}$ & $\begin{array}{l}r^{2} \\
N_{\text {seg }}- \\
\text { flow }\end{array}$ & $\begin{array}{l}\text { Slope } \\
N_{\text {seg }}- \\
\text { vol } \times 10^{-5} \\
(\mu \mathrm{l})\end{array}$ & $\begin{array}{l}r^{2} \\
N_{\text {seg }}- \\
\text { vol }\end{array}$ \\
\hline$R$ & $R$ & $45,800 \pm 19,800$ & $25.2 \pm 9.4$ & $4.27 \pm 1.87$ & 4.7 & 0.97 & 9.2 & 0.95 \\
\hline 1.0 & $R$ & $12,218 \pm 3,278^{*}, * * *$ & $20.8 \pm 5.2 *$ & $1.64 \pm 0.42 *, * * *$ & $15.9 * *$ & 0.98 & $11.0 *, * * *$ & 0.72 \\
\hline 0.7 & $R$ & $16,035 \pm 5,263^{*}$ & $20.6 \pm 6.3^{*}, * * * *$ & $1.93 \pm 0.66^{*}$ & 11.7 & 0.98 & $11.6^{*}$ & 0.85 \\
\hline 0.58 & $R$ & $30,056 \pm 10,476$ & $23.6 \pm 7.6^{*}$ & $2.94 \pm 1.11$ & 7.2 & 0.98 & $9.9 *$ & 0.88 \\
\hline 0.5 & $R$ & $59,018 \pm 18,678$ & $23.7 \pm 6.6^{*}$ & $4.38 \pm 1.40$ & 3.4 & 0.93 & $7.3^{*}$ & 0.94 \\
\hline 0.48 & $R$ & $85,824 \pm 33,664$ & $24.9 \pm 7.6^{*}, * * * *$ & $5.59 \pm 2.18$ & 2.1 & 0.89 & $6.3 *$ & 0.96 \\
\hline$R$ & 1.0 & $4,109 \pm 37 * *$ & $8.6 \pm 0.2^{* *}$ & $1.52 \pm 0.27 * *$ & - & - & - & - \\
\hline$R$ & 1.1 & $28,637 \pm 2,686$ & $30.4 \pm 2.4$ & $4.19 \pm 0.65$ & $8.4 * *$ & 0.91 & $21.5^{* *}$ & 0.78 \\
\hline$R$ & 1.13 & $69,845 \pm 13,656$ & $48.3 \pm 6.9$ & $7.49 \pm 1.88$ & 4.8 & 0.90 & 13.5 & 0.96 \\
\hline$R$ & 1.15 & $13,7671 \pm 34,641$ & $65.4 \pm 11.0$ & $11.80 \pm 3.49$ & 2.9 & 0.83 & 10.0 & 0.99 \\
\hline
\end{tabular}

Each row gives the means and slopes of 50 trees

$R$ random, $N_{\text {seg }}=N_{\text {segments: }}$ total number of segments, vol volume

Upper row: random symmetry and area growth values; rows 2-6: five different symmetry values $(S)$ and random area growth; rows 7-10: four different area growth values $(A)$ and random symmetry

**Means and slopes belonging to different $A$ values (lower 4 rows) in column are significantly different

$* * *$ Values in column are significantly different except those indicated by *

****Only these two groups are significantly different in this column. Multiple comparison of means and slopes using ANOVA with $95 \%$ confidence intervals

The special nature of the symmetrical branching tree (asterisks in Fig. 2) is underlined by the inflow, which is 3.4 times higher than predicted by the fitted

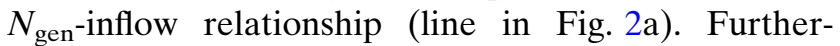
more, $N_{\text {segments }}$ is lower than the lowest number in the random trees. The total volume of the symmetrical branching tree however is close to the regression line.

\subsection{Maximum number of generations and Strahler order in reconstructed trees}

The maximum number of generations, $N_{\text {gen }}$, and the Strahler orders, $N_{\text {strahler }}$, of 50 asymmetrical vascular trees are depicted as function of $N_{\text {segments }}$ in Fig. $2 \mathrm{c}$ and $\mathrm{d}$ (circle). The values for the symmetrical branching tree are shown as well (asterisks). $N_{\text {gen }}$ was weakly 
correlated to $N_{\text {segments }}$ in the stochastic asymmetrical trees $\left(r^{2}=0.23\right)$. Note that $N_{\text {gen }}$ is much smaller in the symmetrical branching tree.

In the stochastic trees, $N_{\text {strahler }}$ vary between 8 and 10 , whereas $N_{\text {strahler }}$ of the symmetric tree is 13 . In the symmetric tree, $N_{\text {strahler }}$ is by definition equal to the $N_{\text {gen }}$.

\subsection{Pressure distribution in reconstructed trees}

The pressure distributions in the trees are depicted in Fig. 3. In the symmetric tree, the pressure drop is mainly located in the distal segments $(<50 \mu \mathrm{m})$, whereas in the asymmetrical branching vascular trees, the pressure drop occurs more gradually; i.e. not only in vascular segments with a diameter $<50 \mu \mathrm{m}$, but also in the vessel segments with larger diameters.

\subsection{Influence of symmetry and area growth on perfusion and tree characteristics}

Total inflow, volume, $N_{\text {gen }}$ and $N_{\text {strahler }}$ as function of $N_{\text {segments }}$ are provided in Fig. 4 for five different symmetry values. For each $S$ value, 50 stochastic trees were generated. For comparison, the values presented in Fig. 2, generated with random $S$ and random $A$ are added (circle). Rows 2-6 of Table 1 summarize the

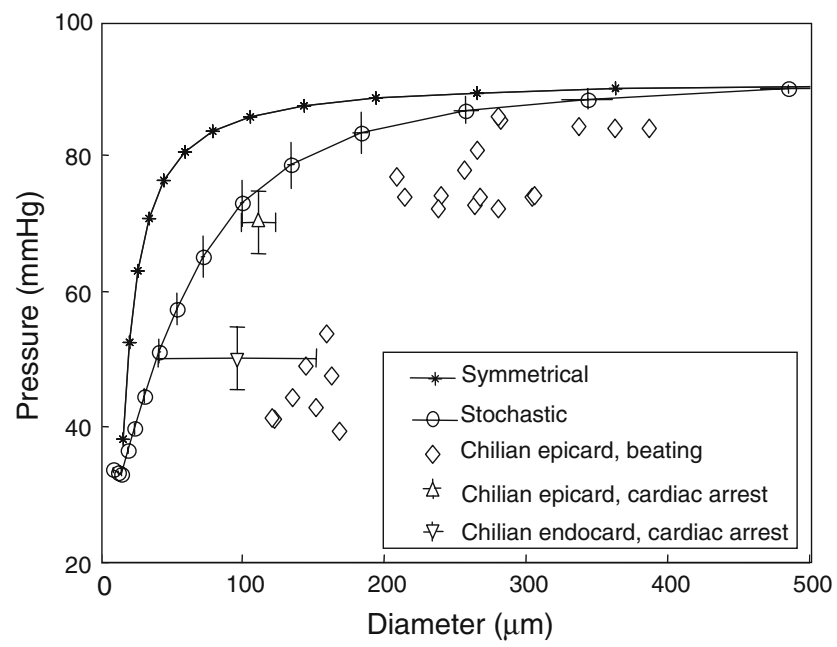

Fig. 3 Pressure distribution. Pressure distribution is given as function of vascular diameter of the stochastic asymmetrical (circle) and the symmetrical branching tree (asterisks). Circle and error bars are mean and standard deviations of pressure and diameter averaged over the 15 diameter classes of the 50 stochastic trees $(n=50)$. Triangles are interpolations of measurements of local pressures in porcine epicardial (downward triangle) and endocardial (upward triangle) arterioles during maximal vasodilation and diastolic arrest [1]. Diamond: measurements in subepicardial large and intermediate arterioles in the maximally vasodilated beating cat heart during diastole data presented in Fig. 4; the smaller the $S$ value the larger the $N_{\text {segments }}$ of the trees. Also the variation in $N_{\text {segments }}$ is larger with smaller $S$.

For each $S$ value, the total inflow values of the 50 trees are linearly related to $N_{\text {segments }}$ in the tree $\left(r^{2}>0.89\right.$, Table 1, Fig. 4a). However, the slopes of these relations are different for each $S$ value (Table 1, ANOVA, $P<0.05$ ). Although $N_{\text {segments }}$ increased with decreasing $S$ values, the ranges of flow in these 50 trees are hardly affected. The mean flow values for $S=0.58$, 0.5 , and 0.48 are not significantly different (Table 1 , ANOVA, $P=0.6)$. Average vascular volume increases with decreasing $S$ (Table 1). The relation between volume and $N_{\text {segments }}$ of all trees presented in Fig. $4 \mathrm{~b}$ is more or less independent of the $S$ value. The bottom panels of Fig. 4 show that at low $S$ values, $N_{\text {gen }}$ is high (Fig. 4c) whereas $N_{\text {strahler }}$ is low (Fig. $4 \mathrm{~d}$ ).

The influence of $A$ values on total inflow, volume, $N_{\text {gen }}$ and $N_{\text {strahler }}$ is provided in Fig. 5. The data presented in Fig. 5 are summarized in row 7-10 of Table 1. $N_{\text {segments }}$ is highly dependent on $A$, which is most clear from Fig. 5c. In case $A$ equals $1, N_{\text {segments }}$ is quite low and independent of $S$ (plus). With increasing $A$, not only $N_{\text {segments }}$ is increasing but also the influence of $S$ as shown by the larger variation in $N_{\text {segments }}$ (Table 1 ). For $A>1$, the total inflow and vascular volume values of the 50 trees are linearly related to $N_{\text {segments }}$ in the tree (Table 1, Fig. 5a, b). The slopes of these relations are also different for different $A$ values (Table 1, ANOVA, $P<0.05)$. $N_{\text {gen }}$ and $N_{\text {strahler }}$ are higher at high $A$ than at low $A$ values.

The $S$ and $A$ values were varied such that $N_{\text {segments }}$ of the trees were $<\sim 200000$, hence having comparable ranges. With the different $S$ values, however, the $N_{\text {gen }}$ in Fig. 4c range between 10 and 170 and $N_{\text {strahler }}$ in Fig. $4 \mathrm{~d}$ range between 6 and 12, whereas with the different $A$ values, these values range between $25-90$ and 7-11, respectively (Fig. 5c, d). Hence these tree characteristics are more sensitive to $S$ than to $A$.

The dependency of pressure distribution on $S$ and $A$ values is depicted in Fig. 6. The pressure distributions for $A=1$ and $S=1$ (plus) are comparable to the nonstochastic symmetrical tree (asterisks). The trees with low $S$ or high $A$ values have a more gradual pressure distribution.

\section{Discussion}

This study shows how different branching rules applied to arterial trees affect perfusion parameters. The trees generated with random symmetry and area growth show large variations in $N_{\text {segments. }}$ Tree size correlates 
Fig. 4 Effect of different constant symmetry values and random area growth values on perfusion and tree characteristics. a Total inflow, b total volume, c maximum number of generations with regions for different $S$ values indicated, and d Strahler order, given as function of $N_{\text {segments }}$ in the trees. Symmetry values are-circle: random (see also Fig. 2), plus: $S=1$, square: $S=0.70$, cross: $S=0.58$, downward triangle: $S=0.5$, diamond: $S=0.48$, area growth values are random. Asterisks: symmetrically branching tree
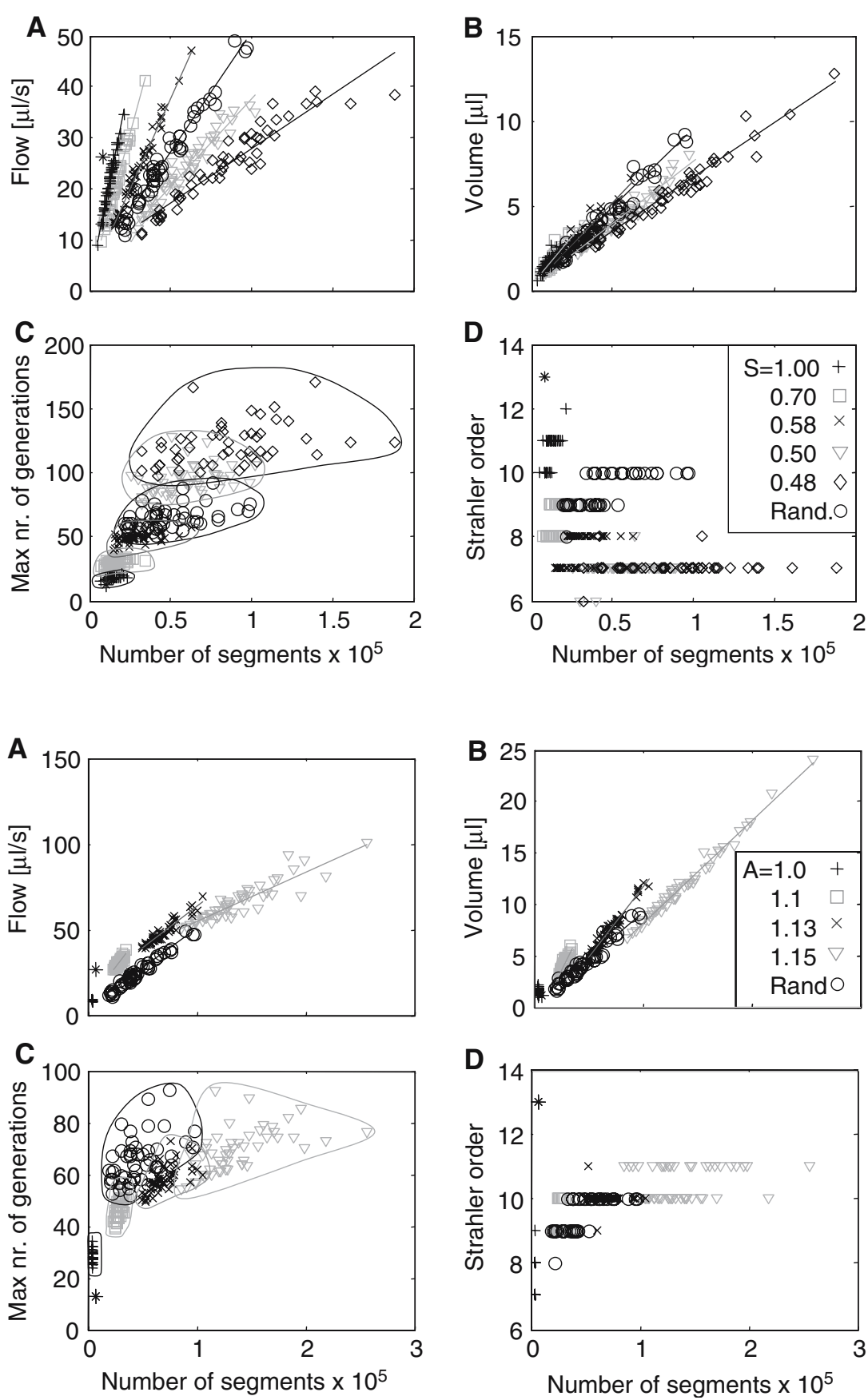

Fig. 5 Effect of different constant area growth values and random symmetry values on perfusion and tree characteristics. a Total inflow, b total volume, c maximum number of generations with regions for different $A$ values indicated, and d Strahler order, given as function of $N_{\text {segments }}$ in the trees. Area growth values are: circle: random (see also Fig. 2), plus: $A=1$, open square: $A=1.1$, cross: $A=1.13$. Downward triangle: $A=1.15$, symmetry values are random. Asterisks: symmetrically branching tree

\subsection{Limitations of the model}

well with both inflow and vascular volume. Area growth in branching nodes strongly affects $N_{\text {segments, }}$ level of inflow and vascular volume. Similarly, symmetry has a strong influence on $N_{\text {segments }}$ and vascular volume, but the average and range of variation of inflow are not affected. The symmetry has more influence on the maximum number of generations than area growth. Stochastic asymmetrical trees having low symmetry and high area growth are characterized by more gradual pressure drops than non-stochastic symmetrical trees.
The process of branching in the trees was continued until vessels smaller than $15 \mu \mathrm{m}$ were generated. Applying smaller diameters for the end segments was not feasible due to limitations in computer hardware. This was especially the case for the large $A$ and small $S$ values. For similar reasons, $S$ values smaller than 0.48 and $A$ values larger than 1.15 were not used. These values are in the range found by Zamir et al. [17]. They found average area ratio values of 1.06 in rat and 1.14 

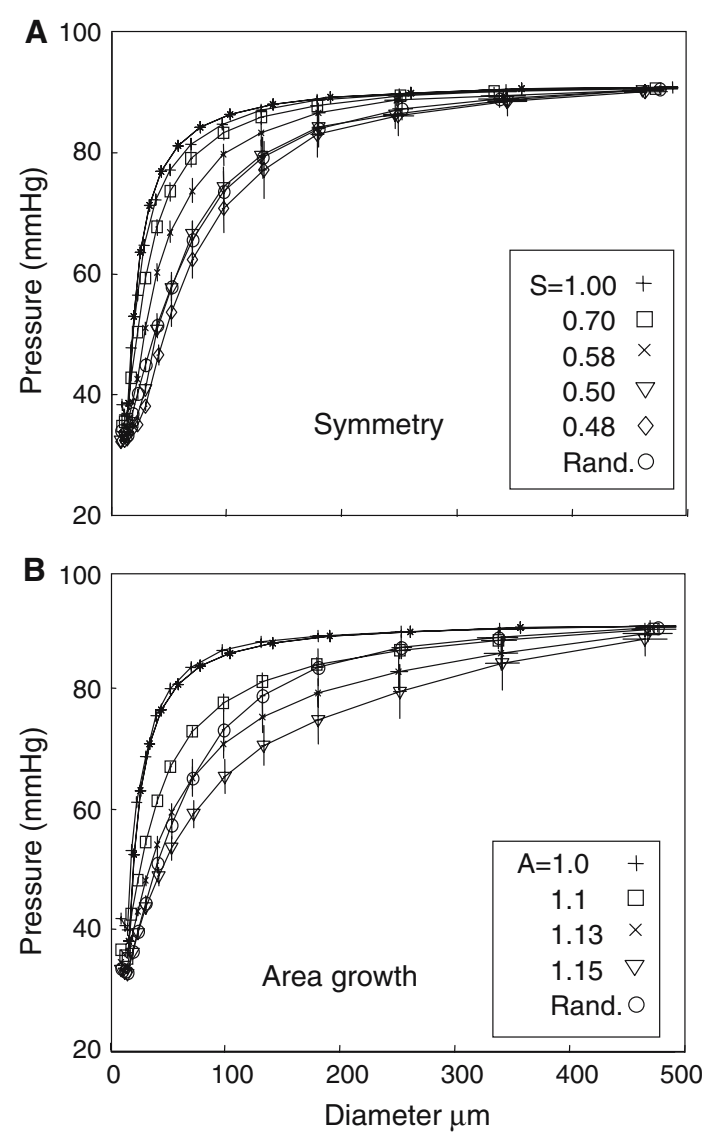

Fig. 6 Influence of symmetry and area growth on pressure distribution. Pressure distribution of stochastic trees with different symmetry values (a) and area growth values (b). Circle: random, asterisks: symmetrical branching tree. $A$ area growth values are: circle: random (see also Fig. 3 ), plus: $A=1$, square: $A=1.1$, cross: $A=1.13$. Downward triangle: $A=1.15$, and symmetry values random. b Symmetry values are: circle: random, plus: $S=1$, square: $S=0.70$, cross: $S=0.58$, downward triangle: $S=0.5$, diamond: $S=0.48$, and area growth values random

for the human coronary bed. Only bifurcations were used in this study because trifurcations are rarely observed. Kassab et al. [6] and Spaan [11] reported that the coronary arterial tree consists of 98 or $99 \%$ bifurcations, respectively. For simplicity, collaterals were also neglected in this study.

For modeling purposes, the fitted distributions shown in the right panels of Fig. 1 were used instead of the measured distribution. Furthermore, the anatomical data provided by VanBavel and Spaan [15] are based on relatively more large vessels than small vessels. In the reconstruction method used here, the distributions of the deviations between fitted and observed values for length and area ratio were assumed not to be dependent on the diameter of the vessel segment. Hence, it is assumed that the distributions provided in right panels of Fig. 1 are representative for the large as well as the small vessels. Kassab et al. [6] measured more segments than VanBavel and Spaan [15]. However, Kassab's classification method, based on a Strahler ordering and a connectivity matrix, is less transparent and was therefore not used in this study.

\subsection{Deterministic versus stochastic trees}

In many studies, symmetric branching is implicitly or explicitly assumed in interpreting data. The advantage of an assumption of symmetry is that, e.g., pressure distribution over the tree can simply be described by a chain of resistances, each resistance having the equivalent magnitude of the vessels of certain order in parallel $[3,12]$. Our results show that the predicted inflow and also the pressure distribution in such symmetric trees differ from those in stochastic branching trees. Attention must therefore be paid to the variation in anatomical parameters, since this variation influences the prediction of local pressures along the bed. This was also shown in model studies performed by VanBavel and Spaan [15] and Dawant et al. [4]. Pries et al. [8] emphasized the importance of variation as an inherent and functionally significant property of microvascular networks, which is not adequately reflected by the typical deterministic branching approach. For example, an estimate of the mean transit time based on averaged quantities of number of generations, segment length and flow velocity is $6.5 \mathrm{~s}$, which is about $60 \%$ higher than the true value in the rat mesentery network (4.08 s). Pries et al. concluded that simplified models of the vascular bed may be inadequate. Therefore stochastic models are necessary to describe functional properties of the microcirculation. It would be interesting to calculate mean transit times in our trees, however, because the outflow system is lacking, this is not possible.

The area ratio is chosen to vary between 1.0 and 1.15. According to Murray's law this should change 1.26. However, the 1.26 is only correct for a purely symmetric node, the 'area ratio' model and the power law model have different mathematical forms, and for non-symmetric branching the area ratio of a 'Murray node' is smaller. More importantly, in the coronary circulation Murray only seems to hold for the smaller arterioles. It has been established (see e.g. [15]) from a.o. arteriolar density and other casting studies that nodes in the larger coronary vessels fulfill a power law with exponent below 3, or smaller area growth (both models equally well fit the available data); this culminates in a lack of area growth in the large epicardial vessels, where velocity rather than shear stress is constant, the power is 2 and $A$ is 1 . Thus, apart from the 
VanBavel study, area ratio values around 1.06 (rat) and 1.14 (human) have been found by Zamir [17].

\subsection{Symmetry $S$ and area growth $A$}

The predicted inflow values are influenced by the tree structure parameters, as is demonstrated in Figs. 4a and 5a. The flow is the result of the pressure drop, which is fixed, and the equivalent resistance of the tree as a whole. In general, resistances in parallel result in reduction of equivalent resistance and resistances in series to an increase in equivalent resistance.

Asymmetrical trees (low $S$ values) are larger than symmetrical trees. Due to the repetitive asymmetrical branching long paths of segments connected in series develop, resulting in high $N_{\text {gen }}$ and high $N_{\text {segments }}$ values. $N_{\text {strahler }}$ is low because the Strahler orders of the large daughter and mother segment are often equally interesting though, symmetry hardly affects the range of inflows of the generated trees (Table 1 for $S=1$, $0.58,0.5,0.48$ and random). Due to short side branches having low resistance connected in parallel at low symmetry, the total resistance of the tree has not been increased. The observation that symmetry affects $N_{\text {gen }}$ and $N_{\text {strhaler, }}$ without affecting inflow indicates that neither $N_{\text {gen }}$ nor $N_{\text {strahler }}$ have a predictive value for the resistance of the vascular bed.

Although symmetry did not influence inflow, area growth does (Table 1). High area growth results in large trees with large branches connected parallel having low total resistance. This influence of area growth becomes more important at low symmetry values, because at low symmetry values larger variations in $N_{\text {segments }}$ are found. Similarly, the influence of symmetry becomes more important at high area growth values. It is interesting to note that choosing only one of the two parameters, $S$ or $A$, random is enough to produce reasonable variations in $N_{\text {segments, }}$ inflow and volume values (Table 1). However, when both symmetry and constant area growth are constant, however, the results are far of from the results with random values (Figs. 4, 5a, b), showing clearly the prerequisite of stochastic tree models in the prediction of inflow and vascular volume values.

It would seem obvious to relate the amount of perfused tissue to the number of terminal arterioles assuming a fixed volume per arteriole. However, Rodriguez-Porcel et al. [10] found an increase in number of smaller arteriolar vessels per $\mathrm{cm}^{3}$ after hypercholesterolemia, while the number of larger arterioles had decreased. The changed branching characteristics were therefore accompanied by changes in amount of tissue that is perfused per end segment. It is therefore difficult to relate the values such as number of end segments and flow through the end segments to the weight of perfused tissue.

\subsection{Pressure distribution in the deterministic trees}

The predictions of the pressure profile along the symmetrical branching tree with vessels $<500 \mu \mathrm{m}$ differs from those in the stochastic trees. The symmetrical tree predicts that the major pressure drop occurs over vessels with a diameter less than $50 \mu \mathrm{m}$. The experimental findings of Chilian et al. [1,2] have been incorporated in Fig. 2. The triangles are measurements of local pressures in porcine epicardial (downward triangle) and endocardial (upward triangle) arterioles during maximal vasodilation and diastolic arrest. The diamonds are measurements in subepicardial large and intermediate arterioles in the maximally vasodilated beating cat heart during diastole. The experimental pressure distribution is more gradual than the distributions in the stochastic trees (Fig. 6). However, Fig. 6 also demonstrates that the branching rules do have a considerable influence on the predicted pressure distribution and that this distribution is more gradual with low symmetry and large area growth values. The discrepancies between the symmetric tree and the stochastic trees underline the fact that asymmetry in the coronary arterial tree cannot be ignored.

\section{Conclusions}

The flow, arterial volume and distribution of pressure, clearly depend on the branching rules applied. The resistance of the symmetrical branching tree is lower and more dominant in the distal part of the tree, arterial volume is smaller. Thus, this study demonstrates that the symmetrical branching arterial tree should be used with caution. Considering the large variations of minimum coronary resistance in perfusion areas within and in between hearts one may assume that there are no strict branching rules for the real coronary tree. Hence, the variation in tree realizations found in the present study with random symmetry and area growth values may very well reflect the biological variation tree anatomies.

\section{References}

1. Chilian WM (1991) Microvascular pressures and resistances in the left ventricular subepicardium and subendocardium. Circ Res 69:561-570 
2. Chilian WM, Layne SM, Klausner EC, Eastham CL, Marcus ML (1989) Redistribution of coronary microvascular resistance produced by dipuridamole. Am J Physiol 256:H383H390

3. Cornelissen AJM, Dankelman J, VanBavel E, Stassen HG, Spaan JA (2000) Myogenic reactivity and resistance distribution in the coronary arterial tree: a model study. Am J Physiol 278:H1490-H1499

4. Dawant B, Levin M, Popel AS (1986) Effect of dispersion of vessel diameters and lengths in stochastic networks. Microvasc Res 31:203-222

5. Kaimovitz B, Lanir Y, Kassab GS (2005) Large-scale 3-D geometric reconstruction of the porcine coronary arterial vasculature based on detailed anatomical data. Ann Biomed Eng 33(11):1517-1535

6. Kassab GS, Rider CA, Tang NJ, Fung Y-CB (1993) Morphometry of pig coronary arterial trees. Am J Physiol Heart Circ Physiol 265:H350-H365

7. Meuwissen M, Chamuleau SA, Siebes M, Schotborgh CE, Koch KT, de Winter RJ, Bax M, de Jong A, Spaan JAE, Piek JJ (2001) Role of variability in microvascular resistance on fractional flow reserve and coronary blood flow velocity reserve in intermediate coronary lesions. Circulation 103:184187

8. Pries AR, Secomb TW, Gessner T, Sperandio MB, Gross JF, Gaehtgens P (1994) Resistance to blood flow in microvessels in vivo. Circ Res 75:904-915

9. Pries AR, Secomb TW, Gaehtgens P (1995) Structure and hemodynamics of microvascular networks: heterogeneity and correlations. Am J Physiol 269:H1713-H1722
10. Rodriguez-Porcel M, Lerman A, Ritman EL, Wilson SH, Best PJM, Lerman LO (2002) Altered myocardial microvascular 3D architecture in experimental hypercholesterolemia. Circulation 102:2028-2030

11. Spaan JAE (1991) Coronary blood flow. Mechanics, distribution, and control. Kluwer, Dordrecht

12. Spaan JAE, Cornelissen AJM, Chan CC, Dankelman J, Yin FCP (2000) The dynamics of flow, resistance and intramural vascular volume in the canine coronary circulation. Am J Physiol 278:H383-H403

13. Tomanek RJ (1987) Microanatomy of the coronary circulation. In: Spaan JAE, Bruschke AVG, Grittenberg-DeGroot AC (eds) Coronary circulation, from basic mechanisms to clinical applications. Martinus Nijhoff, Dordrecht

14. Toyota E, Fujimoto K, Ogasawara Y, Kajita T, Shigeto F, Matsumoto T, Goto M, Kajiya F (2002) Dynamic changes in three-dimensional architecture and vascular volume of transmural coronary microvasculature between diastolicand systolic-arrested rat hearts. Circulation 105:621-626

15. VanBavel E, Spaan JAE (1992) Branching patterns in the porcine coronary arterial tree: estimation of flow heterogeneity. Circ Res 71:1200-1212

16. Vergroesen I, Noble MIM, Wieringa PA, Spaan JAE (1987) Quatification of $\mathrm{O}_{2}$ consumption and arterial pressure as dependent determinants of coronary flow. Am J Physiol 252:H545-H553

17. Zamir M (1988) Distributing and delivering vessels of the human heart. J Gen Physiol 91:725-735 